Revista de

Contabilidade e

Organizações

www.rco.usp.br
DOI: http://dx.doi.org/10.11606/issn.1982-6486.rco.2019.163596

Journal of

Accounting and

Organizations

\title{
Como o volume de negociação reage ao sentimento do investidor?
}

How does trading volume respond to investor sentiment?

Paulo Fernando Marschnera , Paulo Sergio Ceretta ${ }^{a}$

${ }^{a}$ Universidade Federal de Santa Maria

Palavras-chave

Sentimento do investidor.

Mercado de ações.

Finanças comportamentais.
Keywords

Investor sentiment.

Stock market.

Behavioral finance.
Informações do artigo

Recebido: 28 de outubro de 2019

Aprovado: 23 de dezembro de 2019

Publicado: 26 de dezembro 2019

\section{Resumo}

A economia comportamental ampliou a forma de entender as negociações no mercado de ações. Investidores com diferentes expectativas de otimismo (ou pessimismo) e confiança tendem a negociar de formas distintas no curto e longo prazo. Além disso, seus vieses comportamentais tendem a ser uma das causas subjacentes da não linearidade e da assimetria existente no mercado de ações, fator que dificulta uma análise precisa dessa relação. Por meio de uma abordagem não linear de atrasos distribuídos autorregressivos assimétricos foram analisadas detalhadamente as conexões não lineares e assimétricas de curto e longo prazo entre o sentimento do investidor e o volume de negociação no mercado americano entre 2004 a 2017. Os resultados, além de confirmarem que os vieses comportamentais influenciam o declínio do volume de negócios, indicam que o volume de negociação reage rapidamente à presença de investidores com menor nível de confiança e que essa relação é profundamente assimétrica no longo prazo. A partir destas evidências é presumível que em momentos de baixa liquidez a confiança dos investidores decline e sua aversão ao risco aumente, e por isso diminuam suas negociações para evitar resultados negativos.

\begin{abstract}
Behavioral economics has broadened the understanding of stock market trading. Investors with different expectations of optimism (or pessimism) and confidence tend to trade differently over the short and long term. Moreover, their behavioral biases tend to be one of the underlying causes of nonlinearity and stock market asymmetry, a factor that hinders an accurate analysis of this relationship. Using a nonlinear approach to asymmetric autoregressive distributed delays, we analyzed in detail the short and long-term non-linear and asymmetric connections between investor sentiment and trading volume in the US market from 2004 to 2017. The results, besides confirming whereas behavioral biases influence declining turnover, indicate that trading volume reacts rapidly to the presence of lower confidence investors and that this relationship is deeply asymmetrical in the long run. From this evidence it is presumed that in times of low liquidity investor confidence declines and their risk aversion increases, and therefore they reduce their trading to avoid negative results.
\end{abstract}

\section{Implicações práticas}

Agentes do mercado devem considerar a reação do volume de negócios ao sentimento dos investidores, especialmente em períodos de alto otimismo ou confiança excessiva. Com isso, podem redimensionar suas decisões de aumentar ou diminuir seu volume de negociação ou optar por não negociar.

Copyright (C) 2019 FEA-RP/USP. Todos os direitos reservados

Autor correspondente: Tel. +55 (55) 99937-3891

E-mail: paulofernandomarschner@hotmail.com (P. F. Marschner); ceretta10@gmail.com (P. S. Ceretta)

Universidade Federal de Santa Maria. Av. Roraima, 1000 - 4303 - Cidade Universitária - Santa Maria/ RS - 97105-900, Brasil. 


\section{INTRODUÇÃO}

A racionalidade dos agentes econômicos constitui um dos paradigmas centrais da abordagem econômica neoclássica que sustenta a Moderna Teoria de Finanças. Isso implica que todos os agentes que atuam nos mercados financeiros tomam decisões puramente econômicas e são capazes de traduzir corretamente as informações lançadas no mercado. Esse paradigma esteve fortemente vinculado ao conceito de utilidade esperada (Von Neumann \& Morgenstern, 1944) e a hipótese de mercado eficiente (Fama, 1965). Com o rápido crescimento do mercado financeiro, diversos tipos de investidores começaram a participar do mercado de ações e surgiram diversas anomalias financeiras que não estavam em conformidade com o paradigma da racionalidade (Barberis; Shleifer \& Vishny, 1998), indicando que os investidores não atuam de forma racional, pois sua psicologia humana leva a suas decisões de investimento (Akerlof \& Shiller, 2009; Dhaoui, Bourouis \& Boyacioglu, 2013).

Parte considerável dessa literatura foi amparada na teoria do prospecto (Kahneman \& Tversky, 1979) indicando que os indivíduos fazem escolhas diferentes quando existe o risco de ganhos ou perdas, além da falta de total racionalidade. A crescente literatura de finanças comportamentais motivou diversos estudiosos a considerar o sentimento dos investidores no mercado de ações. Baker e Wurgler (2006) criaram um dos mais relevantes índices de sentimento do investidor e descobriram que o padrão de previsibilidade do retorno das ações ao sentimento do investidor varia de acordo com algumas características das ações como o tamanho da empresa, a volatilidade e sua idade. Além deste, diversos trabalhos analisaram a relação entre o sentimento do investidor e o mercado de ações (Kumar \& Lee, 2006; Baker \& Wurgler, 2007; Kaniel, Saar \& Titman, 2008; Yu \& Yuan, 2011; Stambaugh, Yu \& Yuan, 2012; Yu, 2013; Liu, 2015; Debata, Dash \& Mahakud, 2018).

A partir desse contexto, percebe-se que os fenômenos comportamentais do mercado de ações resultantes da irracionalidade dos investidores são extremamente significativos, já que não podem ser explicados pelas principais teorias financeiras clássicas. Embora já existam evidências da relação entre o sentimento do investidor e o mercado de ações, a literatura é inconclusiva, pois espera-se que as expectativas dos investidores mudem de acordo com a chegada de novas informações no mercado (Daniel, Hirshleifer, \& Subrahmanyam, 1998; Kim \& Kim, 2014). Além disso, diversos autores encontraram evidências de que o sentimento do investidor é uma das causas subjacentes da não linearidade e da assimetria no volume de negociação e no preço/retorno das ações (Ding, Charoenwong \& Seetoh, 2004; Schmeling, 2009; Zhang \& Semmler, 2009; Dhaoui \& Bacha, 2017).

De acordo com Ding et al. (2004) e Zhang e Semmler (2009) diferente do sentimento pessimista, o sentimento otimista pode levar a um volume de negociação substancial. Como o sentimento de otimismo é reforçado à medida em que mais agentes aderem à uma tendência de mercado (Brown \& Cliff, 2004, 2005), esperase que os impactos do sentimento otimista sejam diferentes do pessimista no longo prazo (Dhaoui \& Bacha, 2017). Nesse contexto, este trabalho procura verificar a relação assimétrica entre o sentimento do investidor e o volume de negociação. Para analisar essa relação empregou-se um modelo não linear de atrasos distribuídos autorregressivos assimétricos (NARDL) proposto por Shin, Yu e Greenwood-Nimmo (2014) que permite uma análise conjunta de não estacionariedade, não linearidade e ainda captura os efeitos assimétricos no curto e longo prazo, superando as limitações apontadas pela literatura (Ding et al., 2004; Brown \& Cliff, 2004; 2005; Zhang \& Semmler, 2009; Dhaoui \& Bacha, 2017).

Os resultados, além de confirmarem que os vieses comportamentais influenciam o declínio do volume de negócios, indicam que o volume de negociação é mais sensível à presença de investidores com menor nível de confiança e que essa relação é profundamente assimétrica no longo prazo. Como as evidências assimétricas sobre a relação entre o sentimento do investidor e o mercado de ações ainda são incipientes (Ni et al. 2015), esta pesquisa contribui com a literatura apresentando novas evidências e possíveis esclarecimentos. Como a percepção dos investidores sobre o volume de negociação está intimamente relacionada com sua percepção de risco, esses resultados são de grande interesse prático, pois sua percepção geralmente afeta a decisão dos investidores em aumentar ou diminuir seu volume de negociação ou optar por não negociar.

\section{REVISÃO DE LITERATURA}

O trabalho seminal de Kahneman e Tversky (1979) crítica o modelo de utilidade esperada como forma de tomada de decisão dos indivíduos, indicando que estes fazem escolhas diferentes quando existe o risco de ganhos ou perdas, além da falta de total racionalidade. Esse trabalho deu origem à teoria do prospecto e, posteriormente, ao campo de finanças comportamentais que tem sido útil ao explicar situações em que a abordagem tradicional falha. 
Inicialmente, as pesquisas de cunho comportamental focaram em anomalias de mercado (De Bondt \& Thaler, 1985; Porteba \& Summers, 1988; Fama \& French, 1988) ou na volatilidade excessiva não ocasionada por fatores inerentes à empresa (Shiller, 1981). Paralelo a isso, surgiram evidências de que esses fenômenos podem ser provocados pela presença de investidores que não são totalmente racionais, já que negociam de acordo com seus sentimentos (De Long, Shleifer, Summer, \& Waldmann, 1990; Shleifer \& Summers, 1990; Lee, Shleifer, \& Thaler, 1991; Daniel et al. 1998; Barberis et al. 1998; Shiller, 2003).

De acordo com Lee et al. (1991), o sentimento do investidor é um componente do preço dos títulos oriundo de expectativas sobre os retornos que não são justificadas pelos seus fundamentos. Já Brown e Cliff (2004; 2005) argumentam que o sentimento do investidor vem da crença subjetiva de seus investidores e pode tanto influenciar a valorização das ações, como causar expectativas tendenciosas, como a propensão a especular, o otimismo ou o pessimismo dos investidores na avaliação real das ações. Estas crenças subjetivas podem ser reflexo de uma reação negativa ou exagerada em relação a notícias boas/ruins lançadas diariamente no mercado de ações.

Existem diversas evidências sobre a relação entre o sentimento do investidor e o mercado de ações (Baker \& Wurgler, 2006; Kumar \& Lee, 2006; Baker \& Wurgler, 2007; Kaniel et al. 2008; Yu \& Yuan, 2011; Stambaugh et al. 2012; Yu, 2013; Liu, 2015; Debata et al. 2018). De acordo com estes autores, o sentimento do investidor é crucial para afetar o retorno das ações devido a limites de arbitragem, choque de demanda inesperado, desequilíbrio específico, funções de referência e utilidade em ganho e perda. Além disso, sabe-se que o sentimento do investidor tende a ser persistente ao longo do tempo, já que o otimismo é reforçado à medida que mais agentes aderem à uma tendência de mercado e, apesar da arbitragem ser capaz de eliminar estratégias lucrativas de curto prazo, não é capaz de corrigir desvios de preços no longo prazo (Brown \& Cliff, 2004, 2005).

Atualmente, os estudos tentam ir além da mera constatação de que o sentimento do investidor afeta ou não o mercado de ações. Diversos autores encontraram evidências de que o sentimento do investidor é uma das causas subjacentes da não linearidade e da assimetria no volume de negociação e no retorno das ações (Ding et al., 2004; Schmeling, 2009; Zhang \& Semmler, 2009; Dhaoui \& Bacha, 2017). Por esse motivo, Dhaoui e Bacha (2017) indicam a relevância de analisar o sentimento do investidor juntamente com dois componentes do mercado de ações: i) o preço das ações, e ii) o volume de negociação. O primeiro permite supervisionar a confiança dos investidores, enquanto o segundo supervisiona a liquidez do mercado de ações.

O volume de negociação é amplamente utilizado com uma proxy para a liquidez do mercado de ações (Grossman \& Miller, 1988) e parece ser sensível ao sentimento do investidor, respondendo assimetricamente ao otimismo e pessimismo (Dhaoui \& Bacha, 2017). Ding et al. (2004) e Zhang e Semmler (2009) encontraram evidências de que, diferente do sentimento pessimista, o sentimento otimista pode levar a um volume de negociação substancial. De acordo com Dhaoui e Bacha (2017), um investidor otimista tente a aumentar seu volume de negociação quando prevê um aumento no preço das ações. Sua expectativa é que esse aumento gere outro aumento e o proporcione ganhos futuros. No entanto, quando os preços caem, o investidor negocia normalmente já que é menos sensível a resultados negativos. Já um investidor pessimista espera que uma redução nos preços das ações seja seguida por reduções adicionais e, para evitar perdas, tendem a reduzir o volume de negociação. Como os investidores em estado pessimista são menos sensíveis a resultados positivos, tendem a negociar normalmente quando antecipam um aumento de preço.

A teoria do prospecto indica que a dor da perda supera o prazer por um ganho equivalente, e devido a isso, os indivíduos tendem a assumir maiores riscos para evitar uma perda. Embora isso pareça implicar que investidores pessimistas tenham maior predisposição a correr riscos, sabe-se que quando o risco aumenta, o pessimismo aumenta, e apenas investidores otimistas tendem a aceitar situações de maior risco (Paraboni, Rigui, Vieira \& Silveira, 2018).

De acordo com De Bondt e Thaler (1995), o principal fator comportamental para entender o quebracabeça da negociação é o excesso de confiança. Sua influência no volume de negociação é compartilhada por vários autores (De Long, Summer \& Waldmann, 1990; Daniel, Hirshleifer \& Subrahmanyam, 2001). De acordo com Daniel et al. (1998), investidores com excesso de confiança superam a precisão de suas próprias informações e ignoram as informações públicas disponíveis. Eles também, superestimam suas habilidades de julgamento e subestimam as habilidades de outras pessoas. 
Alguns estudos mediram a confiança do investidor com base no impacto do preço das ações no volume de negociação (Boynton, Oppenheimer \& Reid, 2009; Ulussever, Yumusak \& Kar, 2011; Dhaoui et al. 2013), onde uma reação positiva no volume de negociação parece ser um efeito de excesso de confiança, e uma resposta negativa, resultado de um impacto insuficiente da confiança (Dhaoui \& Bacha, 2017). Dessa forma, investidores com confiança excessiva tendem a subestimar sua exposição a riscos agindo de forma agressiva e aumentando seu volume de negociação. Já investidores com confiança insuficiente tendem a superestimar sua exposição a riscos reduzindo seu volume de negócios.

Em uma análise da relação entre risco e sentimento nos mercados americano, alemão e chinês, Paraboni et al. (2018) confirmaram empiricamente que em momentos de baixa liquidez a percepção de risco dos investidores aumenta e por isso eles tendem a reduzir o volume de negociação. Por outro lado, em momentos de alta liquidez, há um aumento no volume de negociação e uma consequente diminuição no risco. Embora divergente, um número considerável de artigos relata um impacto positivo e significativo do preço das ações no volume de negócios (Brailsford, 1996; Lee \& Rui, 2002; Mestel, Gurgul \& Majdosz, 2003).

É teoricamente justificável que o volume de negociação seja assimetricamente sensível ao otimismo, pessimismo, e a confiança dos investidores (Beyer \& Guttman, 2011; Dhaoui \& Bacha, 2017). A teoria do prospecto (Kahneman \& Tversky, 1979) fornece uma explicação razoável para essa relação, uma vez que os indivíduos fazem escolhas diferentes quando há risco de ganho ou perda devido a sua racionalidade limitada.

\section{DADOS E MÉTODO}

Os dados utilizados para o desenvolvimento desta pesquisa correspondem as séries de fechamento do preço das ações e volume de negociação do mercado de ações S\&P500, coletados no site Yahoo Finance, e dados da pesquisa da Associação Americana de Investidores Individuais no período de $1^{\text {o }}$ de janeiro de 2004 a 30 de novembro de 2017. A escolha do mercado americano é atribuída além de sua representatividade, à disponibilidade de uma proxy para sentimento do investidor individual.

O sentimento do investidor é calculado como a diferença entre a previsão alta e a baixa do índice S\&P500: Sentimento $=$ Bullish - Bearish e está disponível em frequência semanal. Como o dia de fechamento do indicador de sentimento difere do fechamento semanal do mercado de ações, os dados de preço e volume foram coletados em frequência diária (3.755 observações) e convertidos em frequência semanal no mesmo dia de fechamento do indicador de sentimento. As séries finais ficaram compostas por 727 observações sincronizadas em frequência semanal, todas expressas em logaritmos naturais.

Como destacado, o volume de negociação permite monitorar o otimismo e o pessimismo dos investidores. Um aumento neste indicador pode ser entendido como uma expectativa de otimismo. No entanto, uma diminuição é entendida como uma expectativa de pessimismo do investidor. Já o preço das ações permite analisar o impacto da confiança do investidor na liquidez do mercado de ações, um impacto positivo do preço das ações no volume de negócios é considerado um indicador de confiança do investidor, caso contrário, é considerado como um indicador de confiança insuficiente (Dhaoui \& Bacha, 2017).

\subsection{Modelo NARDL}

A relação entre séries temporais é geralmente examinada através das técnicas de cointegração, modelagem de correção de erros e causalidade de Granger. No entanto, essas técnicas permitem avaliar suas interações apenas no curto prazo e assumem relações simétricas. Todavia, séries temporais podem ocultar a cointegração se seus componentes positivos e negativos forem cointegrados entre si (Granger \& Yoon, 2002) fragilizando os modelos lineares e simétricos.

Para superar essas limitações Shin et al. (2014) propuseram uma abordagem não linear de cointegração ARDL (NARDL) como uma extensão ao modelo ARDL (Pesaran \& Shin, 1999; Pesaran, Shin \& Smith, 2001), que utiliza decomposições de soma parcial positiva e negativa, permitindo a detecção de efeitos assimétricos de curto e longo prazo. O modelo NARDL apresenta vantagens sobre os modelos clássicos de cointegração. Além de determinar facilmente as relações de cointegração de pequenas amostras (Romilly, Song \& Liu, 2001), pode ser usado mesmo se os regressores estiverem estacionários em nível ou em primeira diferença, isto é I (0) ou I (1). No entanto, não pode ser aplicado se os regressores forem I (2). Para analisar a relação entre o sentimento do investidor e o mercado de ações foi delineado o seguinte modelo: 


$$
V o l_{t}=\alpha_{0}+\alpha_{1} r t_{t}^{+}+\alpha_{2} r t_{t}^{-}+\alpha_{3} \operatorname{sent}_{t}^{+}+\alpha_{4} \operatorname{sent}_{t}^{-}+e_{\mathrm{t}}
$$

Em que, $V o l$ é o volume de negociação, $r t$ é o preço das ações, e sent é o sentimento do investidor; $\alpha\left(\alpha_{0}\right.$, $\left.\alpha_{1}, \alpha_{2}, \alpha_{3}, \alpha_{4}\right)$ é um vetor de parâmetros de longo prazo a ser estimado. Na equação (1) $r t_{t}^{+}$e $r t_{t}^{-}$representam as somas parciais de mudanças positivas e negativas no preço das ações, calculadas da seguinte forma:

$$
\begin{aligned}
& r t_{t}^{+}=\sum_{i=1}^{t} \Delta r t_{t}^{+}=\sum_{i=0}{ }^{t} \max \left(\Delta r t_{i}, 0\right) \\
& r t_{t}^{-}=\sum_{i=1}{ }^{t} \Delta r t_{t}^{-}=\sum_{i=0}{ }^{t} \min \left(\Delta r t_{i^{\prime}}, 0\right)
\end{aligned}
$$

Da mesma forma, sent $t_{t}^{+}$e sent $t_{t}^{-}$são somas parciais de mudanças positivas e negativas no sentimento do investidor, calculadas da seguinte forma:

$$
\begin{gathered}
\operatorname{sent}_{t}^{+}=\sum_{i=1}{ }^{t} \Delta \operatorname{sen} t_{t}^{+}=\sum_{i=1}{ }^{t} \max \left(\Delta \operatorname{sent}_{i}, 0\right) \\
\operatorname{sent}_{t}^{-}=\sum_{i=1}{ }^{t} \Delta \operatorname{sent}_{t}^{-}=\sum_{i=1}{ }^{t} \min \left(\Delta \operatorname{sent}_{i}, 0\right)
\end{gathered}
$$

$\mathrm{Na}$ equação (1) $\alpha_{1}$ e $\alpha_{2}$ capturam a relação de longo prazo entre o volume de negociação e mudanças positivas e negativas nos preços das ações, e $\alpha_{3}$ e $\alpha_{4}$ a relação de longo prazo entre o volume de negociação e mudanças positivas e negativas no sentimento do investidor. A equação (1) pode ser integrada em uma configuração $A R D L$, da seguinte maneira:

$$
\begin{gathered}
\Delta \operatorname{Vol}_{t}=\alpha+\beta_{0} \text { vol }_{t-1}+\beta_{1} r t_{t-1}{ }^{+}+\beta_{2} r t_{t-1}^{-}+\beta_{3} \operatorname{sent}_{t-1}{ }^{+}+\beta_{4} \operatorname{sent}_{t-1}^{-}+\sum_{i=1}^{p} \Delta \theta v o l_{t-1}+\sum_{i=0}^{q}\left(y_{i}^{+} \Delta r t_{t-i}{ }^{+}+y_{i}{ }^{-} \Delta r t_{t-i}{ }^{-}\right) \\
+\sum_{i=0}{ }^{s}\left(\theta_{i}^{+} \Delta \operatorname{sent}_{t-1}{ }^{+}+\theta_{i}^{-} \Delta \operatorname{sent}_{t-i}{ }^{-}\right)+u_{t}
\end{gathered}
$$

Em (6) todas as variáveis são definidas, p e s são ordens de atraso, $\alpha_{1}=\beta_{1} / \beta_{0}, \alpha_{2}=\beta_{2} / \beta_{0}$, são os impactos a longo prazo do aumento e diminuição do preço das ações no volume negociado. Da mesma forma $\alpha_{3}=\beta_{3} / \beta_{0}, \alpha_{4}=$ $\beta_{4} / \beta_{0}$, são os impactos a longo prazo do sentimento dos investidores (otimismo e pessimismo). Nesta configuração, além da relação assimétrica de longo prazo, as influências assimétricas de curto prazo entre as variáveis também são capturadas. $\sum_{i=0}^{q} y_{i}^{+}$e $\sum_{i=0}^{q} y_{i}^{-}$medem os impactos de curto prazo do aumento e diminuição do preço das ações no volume negociado, e $\sum_{i=0} s \theta_{i}^{+}$e $\sum_{i=0} s \theta_{i}^{-}$, os impactos de curto prazo do estado otimista e pessimista do investidor no volume de negócios.

Como feito em trabalhos anteriores (Ibrahim, 2015; Dhaoui \& Bacha, 2017), foram adotadas as seguintes etapas para a implementação desse modelo. Inicialmente, serão aplicados os testes Augmented Dickey-Fuller (1981) e Kwiatkowski-Phillips-Schmidt-Shin (1992) para estabelecer as ordens de integração das variáveis. Apesar do modelo comportar variáveis I (0) ou I (1) é necessário que nenhuma variável I (2) esteja incluída, pois sua presença torna as estatísticas F calculadas para testar a cointegração inválidas. Após confirmar a ausência de variáveis I (2) o modelo (6) será estimado usando o método Ordinary Least Squares padrão. Nesta etapa, será aplicado o procedimento geral ao específico para chegar à especificação final do modelo NARDL. Ou seja, será executado o modelo básico considerando os atrasos ótimos e excluída após cada estimativa os atrasos insignificantes, mantendo apenas resultados significativos para as variáveis independentes.

Na terceira etapa, é testada a presença de cointegração entre as variáveis usando a abordagem desenvolvida por Pesaran et al. (2001) e Shin, et al. (2014). Para tal, testa-se a hipótese nula de que $\beta_{0}=\beta_{1}=\beta_{2}=\beta_{3}=\beta_{4}$ $=0$ usando o teste Wald F. No quarto e último estágio, com a presença de cointegração, serão derivados os efeitos multiplicadores dinâmicos cumulativos assimétricos de aumento de $1 \%$ e redução de $1 \%$ em cada variável independente na variável dependente. O multiplicador cumulativo assimétrico no preço das ações pode ser calculado da seguinte forma: 


$$
\begin{aligned}
& m_{i n}{ }^{+}(r t)=\sum_{i=0}^{n}\left(\partial v o l_{t+i}\right) /\left(\partial r t_{i t-1}{ }^{+}\right), n=0,1,2, \ldots \\
& m_{i n}{ }^{-}(r t)=\sum_{i=0}^{n}\left(\partial v o l_{t+i}\right) /\left(\partial r t_{i t-1}{ }^{-}\right), n=0,1,2, \ldots
\end{aligned}
$$

Da mesma forma, o multiplicador cumulativo assimétrico do indicador de sentimento do investidor pode ser calculado da seguinte forma:

$$
\begin{aligned}
& m_{i n}{ }^{+}(\operatorname{sen} t)=\sum_{i=0}{ }^{n}\left(\partial v l_{t+i}\right) /\left(\partial \operatorname{sent}_{i t-1}{ }^{+}\right), n=0,1,2, \ldots \\
& m_{\text {in }}{ }^{-}(\text {sent })=\sum_{i=0}^{n}\left(\partial v^{\prime} l_{t+i}\right) /\left(\partial \text { sent }_{i t-1}{ }^{-}\right), n=0,1,2, \ldots
\end{aligned}
$$

\section{RESULTADOS E DISCUSSÕES}

Na Tabela 1, são apresentadas as estatísticas descritivas, os testes de estacionariedade e raiz unitária e o teste de cointegração não linear. É possível observar que todas as variáveis em primeira diferença apresentam médias muito próximas. As variáveis do mercado acionário (volume e preço) apresentaram uma distribuição assimétrica e leptocúrtica devido aos valores associados à assimetria e curtose. Essas características são típicas em séries precificadas ou índices de ações. Os valores de mínimo e máximo da variável de sentimento do investidor indicam que embora alguns investidores tenham apresentado um sentimento negativo $(-0,425)$, outros apresentaram um elevado sentimento positivo $(0,508)$, evidenciando na prática indivíduos com diferentes níveis de sentimento negociando no mercado de ações.

Para verificar a presença de variáveis I(2), foram aplicados os testes descritos na seção 3.1 e constatado que todas as variáveis são I(0) e I(1). Após essa etapa a equação (1) foi estimada pelo procedimento geral ao específico. A ordem de atraso máximo considerada foi 4, amparada no critério de informação de Akaike (1978), idêntica a encontrada por Dhaoui e Bacha (2017). Na sequência, foram estimados os testes de limites para cointegração não linear. Os limites das estatísticas $\mathrm{F}$ indicam que o volume de negociação, o preço das ações e o sentimento do investidor são significativos e se acumulam no longo prazo. Com base nesse resultado, é possível analisar a relação entre o volume de negociação com mudanças positivas e negativas nos preços das ações e no sentimento

\begin{tabular}{|c|c|c|c|c|c|c|}
\hline \multirow{2}{*}{ Estatísticas descritivas } & \multicolumn{3}{|c|}{ Nível } & \multicolumn{3}{|c|}{ Diferença } \\
\hline & Volume (ln) & Preço $(\ln )$ & Sentimento & Volume $(\ln )$ & Preço $(\ln )$ & Sentimento \\
\hline Média & 21,940 & 7,282 & 0,047 & 0,001 & 0,001 & $-0,001$ \\
\hline Mediana & 21,999 & 7,219 & 0,051 & $-0,006$ & 0,003 & $-0,002$ \\
\hline Mínimo & 20,537 & 6,526 & $-0,514$ & $-1,793$ & $-0,203$ & $-0,425$ \\
\hline Máximo & 23,086 & 7,858 & 0,561 & 1,267 & 0,116 & 0,508 \\
\hline Desvio Padrão & 0,407 & 0,276 & 0,162 & 0,218 & 0,023 & 0,134 \\
\hline Assimetria & $-0,538$ & 0,231 & $-0,078$ & $-0,380$ & $-2,091$ & 0,093 \\
\hline Curtose & 0,466 & $-0,699$ & 0,112 & 10,226 & 17,812 & 0,311 \\
\hline \multicolumn{7}{|c|}{ Estacionariedade e raiz unitária } \\
\hline $\begin{array}{l}\text { Kwiatkowski-Phillips- } \\
\text { Schmidt-Shin }\end{array}$ & 4,427 & 9,529 & 0,591 & 0,033 & 0,182 & 0,017 \\
\hline p-valor & $<0,01$ & $<0,01$ & 0,032 & $>0,100$ & $>0,100$ & $>0,100$ \\
\hline $\begin{array}{l}\text { Augmented Dickey- } \\
\text { Fuller }\end{array}$ & $-1,405$ & 0,828 & $-1,716$ & $-37,449$ & $-14,519$ & $-34,784$ \\
\hline p-valor & 0,149 & 0,890 & 0,082 & 0,000 & 0,000 & 0,000 \\
\hline \multicolumn{7}{|c|}{ Teste para cointegração não linear } \\
\hline & \multicolumn{3}{|c|}{$F$-statistic } & \multicolumn{2}{|c|}{$\mathrm{p}$-valor } & Conclusão \\
\hline Bounds test & \multicolumn{3}{|c|}{26,34} & \multicolumn{2}{|c|}{0,000} & Cointegração \\
\hline
\end{tabular}
dos investidores.

Tabela 1. Estatísticas descritivas, testes de estacionariedade e raiz unitária e teste de cointegração não linear (período de janeiro de 2004 a novembro de 2017, dados semanais)

Fonte: Elaborada pelos autores. 
Na Tabela 2, é possível observar as estimativas do modelo NARDL para cada umas variáveis, suas variações, e partes positivas e negativas. Para testar a adequabilidade destas estimativas, foram realizados alguns testes de diagnóstico no modelo. O valor de $\mathrm{R}^{2}$ é $(0,32)$ o que mostra um poder razoável das variáveis independentes na explicação da variável dependente, valor superior ao encontrado por Dhaoui e Bacha (2017). O teste de BoxLjung indica que os modelos estão livres de autocorrelação, e o teste de Ramsey RESET que a fórmula funcional utilizada é adequada para analisar a relação entre as variáveis.

Tabela 2. Estimativas NARDL entre volume, preço e sentimento

\begin{tabular}{|c|c|c|}
\hline Variáveis & Coeficiente & p-valor \\
\hline Intercepto & 10,031 & 0,000 \\
\hline $\mathrm{L}(\mathrm{v}, 1)$ & $-0,482$ & 0,000 \\
\hline $\mathrm{L}\left(\mathrm{p} \_\mathrm{p}, 1\right)$ & $-0,760$ & 0,000 \\
\hline $\mathrm{L}\left(\mathrm{p} \_\mathrm{n}, 1\right)$ & $-0,286$ & 0,000 \\
\hline $\mathrm{L}\left(\mathrm{s} \_\mathrm{p}, 1\right)$ & $-0,007$ & 0,903 \\
\hline $\mathrm{L}\left(\mathrm{s} \_\mathrm{n}, 1\right)$ & $-0,101$ & 0,057 \\
\hline $\mathrm{L}\left(\mathrm{r} \_\mathrm{p} \_\mathrm{n}, 0: 0\right) 0$ & $-2,674$ & 0,000 \\
\hline $\mathrm{L}\left(\mathrm{r} \_\mathrm{p} \_\mathrm{p}, 0: 0\right) 0$ & $-0,089$ & 0,896 \\
\hline $\mathrm{L}\left(\mathrm{r} \_\mathrm{s} \_\mathrm{n}, 0: 0\right) 0$ & $-0,012$ & 0,909 \\
\hline $\mathrm{L}\left(\mathrm{r} \_\mathrm{s} \_\mathrm{p}, 0: 0\right) 0$ & $-0,044$ & 0,685 \\
\hline $\mathrm{L}\left(\mathrm{r} \_\mathrm{v}, 1: 4\right) 1$ & $-0,129$ & 0,010 \\
\hline $\mathrm{L}\left(\mathrm{r}_{-} \mathrm{v}, 1: 4\right) 2$ & $-0,087$ & 0,061 \\
\hline $\mathrm{L}\left(\mathrm{r} \_\mathrm{v}, 1: 4\right) 3$ & $-0,047$ & 0,265 \\
\hline L(r_v, 1:4)4 & $-0,098$ & 0,007 \\
\hline \multicolumn{3}{|l|}{ Testes de diagnóstico } \\
\hline Adj. $\mathrm{R}^{2}$ & 0,320 & - \\
\hline Box-Ljung test (4) & 0,526 & 0,971 \\
\hline Box-Ljung test (8) & 8,480 & 0,388 \\
\hline Ramsey RESET test & 0,358 & 0,550 \\
\hline
\end{tabular}

Fonte: Elaborada pelos autores.

Na Tabela 3, é possível observar os coeficientes da cointegração de longo prazo. Os resultados indicam que as partes positivas e negativas do preço das ações impactam negativamente o volume negociado, sendo o impacto causado pela parte positiva mais elevado do que a parte negativa. Diferente de trabalhos anteriores que indicam um excesso de confiança dos investidores (Brailsford, 1996; Lee \& Rui, 2002; Mestel et al. 2003; Dhaoui \& Bacha, 2017), estes resultados apontam que os investidores possuem uma confiança insuficiente na liquidez do mercado de ações. Para os investidores pouco confiantes, o declínio é mais provável que um desenvolvimento favorável, por isso eles reagem diminuindo sua oferta (ou demanda) na esperança de evitar perdas intensas, o que se manifesta por uma redução no volume de transações. Consequentemente, e por extensão a esses resultados, é presumível que investidores com confiança insuficiente tenham mais aversão ao risco e diminuam suas negociações quando percebem que o resultado poderá ser negativo (Dhaoui et al. 2013). Esse resultado corrobora com o trabalho de Paraboni et al. (2018) que já havia encontrado evidências de que em momentos de baixa liquidez a percepção de risco dos investidores aumenta e eles tendem a reduzir o volume de negociação.

O sentimento do investidor não apresentou uma relação estatisticamente significativa, indicando que os investidores não alteram seu volume de negociação no longo prazo a partir de suas expectativas de otimismo e pessimismo.

Tabela 3. Coeficientes da relação de longo prazo e p-valor do teste Wald F

\begin{tabular}{lrr}
\hline Variáveis & Coeficiente & p-valor \\
\hline Preço positivo & $-1,575$ & 0,000 \\
Preço negativo & $-0,594$ & 0,000 \\
Sentimento positivo & $-0,014$ & 0,903 \\
Sentimento negativo & $-0,210$ & 0,056 \\
\hline
\end{tabular}

Fonte: Elaborada pelos autores. 
Após esta etapa, para confirmar a existência de uma relação assimétrica entre as variáveis, foi testada a hipótese nula de simetria versus a alternativa assimétrica. Esse teste verifica se os impactos das partes positivas e negativas afetam de forma semelhante o volume de negociação. A partir dos resultados, é possível observar que o preço das ações impacta de forma assimétrica o volume de negociação no curto e longo prazo. Esse resultado pode estar relacionado aos diferentes níveis de confiança e aversão ao risco dos investidores, que os leva a negociarem de diferentes formas.

Já o sentimento do investidor impacta de forma assimétrica o volume de negociação apenas no longo prazo. Brow e Cliff $(2004 ; 2005)$ apresentam uma explicação plausível para tal relação, indicando que o sentimento do investidor tende a ser persiste ao longo do tempo, já que o otimismo é reforçado a medida em que mais agentes aderem a uma tendência de mercado e, apesar da arbitragem ser capaz de eliminar as estratégias lucrativas de curto prazo, não é capaz de corrigir desvios de preços no longo prazo.

Tabela 4. Teste de assimetria (Wald F)

\begin{tabular}{lccc}
\hline Relação & Teste & p-valor & Conclusão \\
\hline Longo prazo & & & \\
\hline Preço $(+=-)$ & 145,500 & 0,000 & Assimetria \\
Sentimento $(+=-)$ & 208,710 & 0,000 & Assimetria \\
\hline Curto prazo & & & \\
\hline Preço (+= -) & 7,181 & 0,008 & Assimetria \\
Sentimento $(+=-)$ & 0,033 & 0,856 & Simetria \\
\hline
\end{tabular}

Fonte: Elaborada pelos autores.

A partir desses resultados, é possível verificar que o volume de negociação, o preço das ações e o sentimento do investidor compartilham uma associação assimétrica de longo prazo. No curto prazo, a relação assimétrica é existente apenas entre o preço das ações e o volume de negociação. A impressão geral é que os investidores menos confiantes superestimam a probabilidade de que maus resultados ocorram e reagem reduzindo o volume de transações na esperança de reduzir suas perdas. Quanto aos investidores otimistas e pessimistas, a impressão é de que suas expectativas sejam compartilhadas por diversos agentes ocasionando uma tendência de mercado cujos desvios de preço são difíceis de corrigir no longo prazo.

Os resultados indicam que o mercado de ações tende a ser impulsionado por expectativas não racionais, corroborando fortemente com os pressupostos de Akerlof e Shiller (2009), que recomendam incorporar aspectos psicológicos para o entendimento do mercado. Os vieses comportamentais influenciam significativamente o declínio do volume de negócios no mercado acionário. Resultados mais específicos indicam que o volume de negociação é mais sensível à presença de investidores com menor nível de confiança, e no longo prazo.

\section{CONCLUSÃO}

Neste artigo, são discutidos alguns fatores comportamentais que podem explicar a mudança no volume de negócios. Para tal, foi analisada a relação entre o volume de negociação, o preço das ações e o sentimento dos investidores no mercado de ações S\&P500. Os resultados indicam que o mercado de ações tende a ser impulsionado por expectativas não racionais já que sua liquidez não está linearmente relacionada ao preço das ações e ao sentimento do investidor. Os vieses comportamentais influenciam significativamente o declínio do volume de negócios e indicam que o volume de negociação é mais sensível à presença de investidores com menor nível de confiança, e no longo prazo. A partir desses resultados, é presumível que em momentos de baixa liquidez a confiança dos investidores decline e sua aversão ao risco aumente, e por isso diminuam suas negociações para evitar resultados negativos, confirmando proposições anteriores (Dhaoui et al. 2013; Dhaoui \& Bacha, 2017; Paraboni et al. 2018).

As implicações teóricas desta pesquisa abrangem aspectos relacionados à forma como os agentes podem visualizar o mercado acionário em diferentes horizontes temporais, revelando assim suas expectativas de otimismo, pessimismo e confiança. Em termos práticos, estes resultados podem ser particularmente interessantes para investidores no seu processo de tomada de decisão em investimentos, já que sua percepção de risco geralmente afeta a decisão de aumentar ou diminuir seu volume de negociação ou optar por não negociar. Além disso, sugerem aos investidores cautela nas negociações em períodos de alto otimismo ou confiança excessiva, já que nesses períodos as informações sobre investimentos podem conter valores majorados que enviesem sua percepção de risco. 
Embora os resultados obtidos sejam consistentes teoricamente e com orientação prática, o alcance do presente estudo é limitado por sua capacidade de generalização em função de considerar apenas o mercado americano. Pesquisas futuras podem aprofundar essa investigação em outros mercados. Além disso, seria promissor analisar os impactos de vieses comportamentais na volatilidade do mercado de ações.

\section{REFERÊNCIAS}

AAII. (2017). American Association of Individual Investors. Recuperado de: https://www.aaii.com/sentimentsurvey (acesso em 10 de dezembro de 2017).

Akaike, H. (1973). Maximum likelihood identification of gaussian autoregressive moving average models. Biometrika, 60, p. 255-265.

Akerlof, G. A., \& Shiller, J. S. (2009). Animal spirits: How human psychology drives the economy, and why it matters for global capitalism. NJ: Princeton University Press.

Baker, M., \& Wurgler, J. (2006). Investor sentiment and cross-section of stock return. The Journal of Finance, 61(4), p. 1645-1680. DOI: https://doi.org/10.1111/j.1540-6261.2006.00885.x.

Baker, M., \& Wurgler, J. (2007). Investor Sentiment in the Stock Market. Journal of Economic Perspectives, 21(2), p. 129-151. DOI: https://doi.org/10.3386/w13189.

Barberis, N., \& Thaler, R. (2002). Survey of behavioral finance. National Bureau of Economic Research, NBER Working Papers.

Barberis, N., Shleifer, A., \& Vishny, R. (1998). A model of investor sentiment. Journal of Financial Economics, 49, p. 307-343.

Beyer, A., \& Guttman, I. (2011). The effect of trading volume on analysts' forecast bias. The Accounting Review, p. 451-481. DOI: https//doi.org/10.2308/accr.00000030.

Boynton, W., Oppenheimer, H., \& Reid, S. (2009). Japanese day-of-the-week returns patterns: New results. Global Finance Journal, p. 1-12.

Brailsford, T. (1996). The empirical relationship between trading volume, returns and volatility. Accounting \& Finance, 36(1), p. 89-111. DOI: https://doi.org/10.1111/j.1467-629X.1996.tb00300.x.

Brown, G., \& Cliff, M. (2004). Investor sentiment and the near-term stock market. Journal of Empirical Finance, 11(1), p. 1-27. DOI: https://doi.org/10.1016/j.jempfin.2002.12.001.

Brown, G., \& Cliff, M. (2005). Investor Sentiment and Asset Valuation. The Journal of Business, 78(2), p. 405440. DOI:https//doi.org/10.1086/427633.

Daniel, K., Hirshleifer, D. A., \& Subrahmanyam, A. (1998). Investor Psychology and Security Market Under- and Overreactions. The Journal of Finance, 53(6), p. 1839-1885. DOI: https://doi.org/10.1111/0022-1082.00077.

Daniel, K., Hirshleifer, D., \& Subrahmanyam, A. (2001). Overconfidence, arbitrage, and equilibrium asset pricing. The Journal of Finance, 56, p. 921-965. DOI: https://doi.org/10.1111/0022-1082.00350.

De Bondt, W., \& Thaler, R. (1985). Does the stock market overreact? The Journal of Finance, 40(3), $793-805$. DOI: https//doi.org/10.2307/2327804.

De Long, J., Shleifer, A., Summer, L., \& Waldmann, R. (1990). Noise trader risk in financial markets. The Journal of Political Economy, 98(4), p. 703-738.

Debata, B., Dash, S, R., Mahakud, J. (2018). Investor sentiment and emerging stock market liquidity. Finance Research Letters, 26, p.15-31. DOI: https://doi.org/10.1016/j.frl.2017.11.006.

Dhaoui, A., \& Bacha, S. (2017). Investor emotional biases and trading volume's asymmetric response: A nonlinear ARDL approach tested in S\&P500 stock market. Cogent economics \& finance, 1(5), p. 1-13. DOI: https://doi.org/10.1080/23322039.2016.1274225.

Dhaoui, A., Bourouis, S., \& Boyacioglu, M. (2013). The impact of investor psychology on stock markets: Evidence from France. Journal of Academic Research in Economics, p. 35-59.

Dickey, D., \& Fuller, W. (1981). Likelihood ratio statistics for autoregressive time series with a unit root. Econometrica, 49(4), p. 1057-1072. 
Ding, D., Charoenwong, K., \& Seetoh, R. (2004). Prospect theory, analyst forecasts, and stock returns. Journal of Multinational Financial Management, 14(4), p. 425-442. DOI: https://doi.org/10.1016/j.mulfin.2004.03.005.

Fama, E. (1965). The behavior of stock-market prices. Journal of Business, 38(1), 34-105.

Fama, E., \& French, K. (1988). Permanent and Temporary Components of Stock Prices. The Journal of Political Economy, 96(2), p. 246-273.

Granger, C., \& Yoon, G. (2002). Hidden cointegration. University of California, Working Paper 2002-02.

Grossman, S., \& Miller, M. (1988). Liquidity and market structure. The Journal of Finance, p. 617-633.

Ibrahim, M. (2015). Oil and food prices in Malaysia: a nonlinear ARDL analysis. Agricultural and Food Economics, 3(2), 1-14. DOI: https://doi.org/10.1186/s40100-014-0020-3.

Kahneman, D., \& Tversky, A. (1979). Prospect theory: an analysis of decision under risk. Econometrica, p. 263291.

Kaniel, R., Saar, G., \& Titman, S. (2008). Individual investor trading and stock returns. The Journal of Finance, 63 , p. $273-310$.

Kim, S.-H., \& Kim, D. (2014). Investor sentiment from internet message posting and the predictability of stock returns. Journal of Economic Behavior Organization, 107(B), p. 708-729. DOI: https://doi.org/10.1016/j. jebo.2014.04.015.

Kumar, A., \& Lee, C. (2006). Retail investor sentiment and return comovements. The Journal of Finance, 61, p. 2451-2486.

Kwiatkowski, D., Phillips, P., Schmidt, Y., \& Shin, T. (1992). Testing the null hypothesis of stationarity against the alternative of a unit root. Journal of Econometrics, 54, p. 159-178.

Lee, B., \& Rui, O. (2002). The dynamic relationship between stock returns and trading volume: Domestic and cross-country evidence. Journal of Banking \& Finance, 26(1), p. 51-78. DOI: https://doi.org/10.1016/S03784266(00)00173-4.

Lee, C., Shleifer, A., \& Thaler, R. (1991). Investor Sentiment and the Closed-End Fund Puzzle. Journal of Finance, 46(1), p. 75-109.

Liu. S. (2015). Investor Sentiment and Stock Market Liquidity. Journal of Behavior Finance, 15(1), p. 51-67. DOI: https://doi.org/10.1080/15427560.2015.1000334.

Mestel, R., Gurgul, H., \& Majdosz, P. (2003). The empirical relationship between stock returns, return volatility and trading volume an the Australian stock market (Research Paper). University of Graz, Institute of Banking and Finance, Graz.

Ni, Z., Wang, D., \& Xue, W. (2015). Investor sentiment and its nonlinear effect on stock returns - New evidence from the Chinese stock market based on panel quantile regression model. Economic Modelling, 50, p. 266274. DOI: https://doi.org/10.1016/j.econmod.2015.07.007.

Paraboni, A. L., Righi, M. B., Vieira, K. M., \& Silveira, V. G. (2018). The Relationship between Sentiment and Risk in Financial Markets. Brazilian Administration Review, 15(1), p. 1-15. DOI: http://dx.doi.org/10.1590/18077692 bar2018170055.

Pesaran, M. H., \& Shin, Y. (1999). An autoregressive distributed lag modeling approach to cointegration analysis. Em S. Strom, Econometrics and Economic Theory in the 20th Century: The Ragnar Frisch Centennial Symposium. Cambridge: Cambridge University Press.

Pesaran, M. H., Shin, Y., \& Smith, R. J. (2001). Bounds testing approaches to the analysis of level relationships. Journal of Applied Econometrics (16), p. 289-326.

Porteba, J., \& Summers, L. (1988). Mean Reversion in Stock Returns: Evidence and Implications. Journal of Financial Economics, 22(1), p. 27-59.

Romilly, P., Song, H., \& Liu, X. (2001). Car ownership and use in Britain: a comparison of the empirical results of alternative cointegration estimation methods and forecasts. Applied Economics, p. 1803-1818.

Schmeling, M. (2009). Investor sentiment and stock returns: some international evidence. Journal of Empirical Finance, 16(3), p. 394-408. DOI:https://doi.org/10.1016/j.jempfin.2009.01.002. 
Shiller, R. (1981). Do Stock Prices Move Too Much to be Justified by Subsequent Changes in Dividends? The American Economic Review, p. 421-436.

Shiller, R. J. (2003). From Efficient Markets Theory to Behavioral Finance. The Journal of Economic Perspectives, 17(1), p. 83-104.

Shin, Y., Yu, B., \& Greenwood-Nimmo, M. (2014). Modelling asymmetric cointegration and dynamic multipliers in a nonlinear ARDL framework. Econometric Methods and Applications, p. 281-314. DOI: https://doi. org/10.1007/978-1-4899-8008-3_9.

Shleifer, A., \& Summers, L. (1990). The noise trader approach to finance. Journal of Economic Perspectives, p. 19-33.

Stambaugh, R., Yu, J., \& Yuan, Y. (2012). The Short of It: Investor Sentiment and Anomalies. Journal of Financial Economics, 104(2), p. 288-302. DOI: https://doi.org/10.1016/j.jfineco.2011.12.001.

Ulussever, T., Yumusak, T., \& Kar, M. (2011). The day-of-the-week effect in the Saudi stock exchange: a nonlinear garch analysis. Journal of Economics and Social Studies, p. 9-23.

Von Neumann, J., \& Morgenstern, O. (1944). Theoru of games and economic behavior. New Jersey: Princeton University Press.

Yahoo Finance. (2017). Business Finance, Stock Market, Quotes, News. Recuperado de: https://finance.yahoo. com/ (acesso em 10 de dezembro de 2017).

Yu, J. (2013). A sentiment-based explanation of the forward premium puzzle. Journal of Monetary Economics, 60(4), p. 474-491. DOI: https://doi.org/10.1016/j.jmoneco.2013.04.001.

Yu, J., \& Yuan, Y. (2011). Investor sentiment and the mean-variance relation. Journal of Financial Economics, 100(2), p. 367-381. DOI: https://doi.org/10.1016/j.jfineco.2010.10.011.

Zhang, W., \& Semmler, W. (2009). Prospect theory for stock markets: Empirical evidence with time-series data. Journal of Economic Behavior and Organization, 72(3), p. 835-849. DOI: https://doi.org/10.1016/j. jebo.2009.08.003.

\section{Como citar este artigo}

Marschner, P. F., \& Ceretta, P. S. (2019). Como o volume de negociação reage ao sentimento do investidor? Revista de Contabilidade e Organizações, 13:e163596. DOI: http://dx.doi.org/10.11606/issn.1982-6486. rco.2019.163596 